\title{
CDISC SDTM Thrombolysis in Myocardial Infarction Flow Responses Terminology
}

National Cancer Institute

\section{Source}

National Cancer Institute. CDISC SDTM Thrombolysis in Myocardial Infarction Flow Responses Terminology. NCI Thesaurus. Code C101841.

Terminology associated with the time flow responses codelist of the Clinical Data Interchange Standards Consortium (CDISC) Study Data T abulation Model (SDT M). 